\title{
BMJ Open Is clopidogrel better than aspirin following breakthrough strokes while on aspirin? A retrospective cohort study
}

\author{
Meng Lee, ${ }^{1}$ Yi-Ling Wu, ${ }^{1}$ Jeffrey L Saver, ${ }^{2}$ Hsuei-Chen Lee, ${ }^{3}$ Jiann-Der Lee, ${ }^{1}$ \\ Ku-Chou Chang, ${ }^{4}$ Chih-Ying Wu, ${ }^{1}$ Tsong-Hai Lee, ${ }^{5}$ Hui-Hsuan Wang, ${ }^{6}$ \\ Neal M Rao, ${ }^{2}$ Bruce Ovbiagele ${ }^{7}$
}

To cite: Lee M, Wu Y-L, Saver JL, et al. Is clopidogrel better than aspirin following breakthrough strokes while on aspirin? A retrospective cohort study. BMJ Open 2014:4:e006672.

doi:10.1136/bmjopen-2014006672

- Prepublication history for this paper is available online. To view these files please visit the journal online (http://dx.doi.org/10.1136/ bmjopen-2014-006672).

Received 23 September 2014 Revised 28 October 2014 Accepted 11 November 2014

CrossMark

For numbered affiliations see end of article.

Correspondence to Professor Bruce Ovbiagele; Ovibes@musc.edu

\section{ABSTRACT}

Objective: There is insufficient evidence on which to base a recommendation for optimal antiplatelet therapy following a stroke while on aspirin. The objective was to compare clopidogrel initiation vs aspirin reinitiation for vascular risk reduction among patients with ischaemic stroke on aspirin at the time of their index stroke.

Design: Retrospective.

Setting: We conducted a nationwide cohort study by retrieving all hospitalised patients ( $\geq 18$ years) with a primary diagnosis of ischaemic stroke between 2003 and 2009 from Taiwan National Health Insurance Research Database.

Participants: Among 3862 patients receiving aspirin before the index ischaemic stroke and receiving either aspirin or clopidogrel after index stroke during follow-up period, 1623 were excluded due to a medication possession ratio $<80 \%$. Also, 355 were excluded due to history of atrial fibrillation, valvular heart disease or coagulopathy. Therefore, 1884 patients were included in our final analysis.

Interventions: Patients were categorised into two groups based on whether clopidogrel or aspirin was prescribed during the follow-up period. Follow-up was from time of the index stroke to admission for recurrent stroke or myocardial infarction, death or the end of 2010. Primary and secondary outcome measures: The primary end point was hospitalisation due to a new-onset major adverse cardiovascular event (MACE: composite of any stroke or myocardial infarction). The leading secondary end point was any recurrent stroke.

Results: Compared to aspirin, clopidogrel was associated with a lower occurrence of future MACE (HR=0.54, $95 \% \mathrm{Cl} 0.43$ to $0.68, p<0.001$, number needed to treat: 8) and recurrent stroke (HR=0.54, $95 \%$ $\mathrm{Cl} 0.42$ to $0.69, p<0.001$, number needed to treat: 9 ) after adjustment of relevant covariates.

Conclusions: Among patients with an ischaemic stroke while taking aspirin, clopidogrel initiation was associated with fewer recurrent vascular events than aspirin reinitiation.

The term 'aspirin resistance' has been used to describe the failure of aspirin to produce an expected response on one or more laboratory
Strengths and limitations of this study

- It is a nationwide cohort study.

- All participants in our cohort received aspirin for more than 30 days with average dose of $101.3 \mathrm{mg} /$ day at the time of the index stroke.

- It is a retrospective cohort study and reasons for using one specific kind of antiplatelet therapy are not well known in this cohort study.

measures of platelet activation and aggregation. ${ }^{1}$ Mechanistic approaches to investigating aspirin resistance have relied mostly on ex vivo evaluations of platelet function. ${ }^{2}$ However, while platelet aggregability is a major contributor to occlusive vascular events, ${ }^{3}$ other factors, such as vascular endothelial dysfunction, ${ }^{4}$ clotting protein cascades ${ }^{5}$ and flow stasis ${ }^{6}$ are also relevant. This multifactorial complexity, along with differing methods for making ex vivo assessments of platelet function, have made linkage between abnormal platelet function on laboratory indices and hard clinical events inconsistent. As a result, defining 'aspirin resistance' primarily based on currently available laboratory measures may not necessarily be the most appropriate way of discriminating people at high risk for future vascular events while on aspirin.

On the other hand, the potentially more clinically relevant term 'aspirin treatment failure' has been used to describe a person who, regardless of laboratory results, experiences a breakthrough ischaemic event, such as stroke, while receiving aspirin. ${ }^{7}$ The diagnosis of aspirin treatment failure is simpler to diagnose on a consistent basis in everyday routine clinical practice. However, the term 'aspirin failure' can be conceptually misleading when recurrent events occur through mechanisms that aspirin is not expected to influence, such as collateral failure, and when the failure is actually due to non- 
adherence to prescribed aspirin rather than pharmacological ineffectiveness. Although alternative antiplatelet agents are often considered, as mentioned in prevailing expert consensus clinical practice guidelines, there is insufficient evidence on which to base a recommendation for optimal antiplatelet therapy following a stroke while on aspirin. ${ }^{8}$

The objective of this study was to compare the effectiveness of clopidogrel vs aspirin for vascular risk reduction among patients with ischaemic stroke who were on aspirin treatment at the time of their index stroke.

\section{METHODS}

\section{Study design and dataset}

We conducted a nationwide cohort study by retrieving all hospitalised patients ( $\geq 18$ years) with a primary diagnosis of ischaemic stroke between 2003 and 2009 from Taiwan National Health Insurance Research Database (NHIRD). Taiwan has launched a compulsory National Health Insurance programme since 1995, which covers $99 \%$ of the population and reimburses for outpatients, inpatient services as well as prescription drugs. All contracted institutions must file claims according to standard formats, which later transform into the NHIRD. The accuracy of diagnosis of major diseases in the NHIRD, such as stroke, has been validated. ${ }^{9}$

\section{Study population}

We identified all hospitalised patients who were admitted with a primary diagnosis of ischaemic stroke (International Classification of Diseases, Ninth Revision (ICD-9) codes 433, 434, 436) among subjects ( $\geq 18$ years) encountered between 2003 and 2009. This is a nationwide study that included all available and eligible patients. We defined the first ischaemic stroke during study period as the index stroke. We retrieved the information of medications prescribed by physicians prior to index stroke among these patients from the pharmacy prescription database. Only patients with ischaemic stroke who received continuous aspirin treatment $\geq 30$ days before the index stroke were included in our study cohort. The Charlson index was used as a measure for overall severity of comorbidities for index stroke. ${ }^{10}$ Comorbidities were confirmed by ICD-9 codes based on the diagnoses of hospitalisation for index stroke. We excluded patients with atrial fibrillation, valvular heart disease or coagulopathy, since anticoagulants, rather than antiplatelet agents, are generally more suitable for secondary stroke prevention among these patients. Information regarding patients' medications during the follow-up period was retrieved from the pharmacy prescription database. Patients were categorised into two groups based on whether clopidogrel or aspirin was prescribed during the follow-up period. Patients were excluded if they switched antiplatelet therapy between aspirin and clopidogrel during the follow-up period to make the analyses straightforward.
The Taiwan National Health Insurance Bureau provides reimbursement for the use of clopidogrel in patients with ischaemic stroke who are allergic to aspirin or have peptic ulcer (the latter confirmed by prior or current pan-endoscopy results). Although 'aspirin treatment failure' is not one of the prespecified criteria for clopidogrel use, the Bureau typically provides reimbursement in these circumstances. As such, physicians generally have broad latitude to prescribe clopidogrel or aspirin based on their personal preferences. Patients were excluded if their medication possession ratio (number of days drug supplied divided by the number of days in the follow-up period) was $<80 \%$ or clopidogrel or aspirin was not prescribed within 30 days before an end point to reduce bias from poor drug adherence or antiplateletdiscontinuation effects. ${ }^{11} 12$

\section{Main outcome measures}

The primary end point was the first event of a new-onset major adverse cardiovascular event (MACE: composite of any stroke (ischaemic or haemorrhagic) or myocardial infarction). The leading secondary end point was the first event of any recurrent stroke (ischaemic or haemorrhagic) alone. Additional secondary end points were ischaemic stroke, intracranial haemorrhage (codes 430-432), fatal stroke, myocardial infarction (code 410) and all-cause mortality. Follow-up was from time of the index stroke to admission for the first event of recurrent stroke (codes $430-434,436$ ) or myocardial infarction, death, or the end of 2010. National Health Insurance is a compulsory programme in Taiwan, and moving out of the country, which is supposed to be scarce among patients with stroke, is almost the only reason, besides death, for being withdrawn from this programme. A previous study from the Taiwan NHIRD also used 'withdrawn' from this programme to define death. ${ }^{13}$ Therefore, we defined death as in-hospital death or withdrawal of the patient from the National Health Insurance programme.

\section{Statistical analysis}

The baseline characteristics of two treatment groups were compared using student $\mathrm{t}$ test for continuous variables and $\chi^{2}$ test for categorical variables. Kaplan-Meier plots were generated, and the log-rank test was used to evaluate the difference between curves. We employed Cox's proportional hazard model to estimate the unadjusted and adjusted HRs and 95\% CIs, which considered the aspirin group as the reference group. The model was adjusted for baseline age, gender, hypertension, diabetes, prior stroke, prior ischaemic heart disease, hyperlipidaemia, gastrointestinal bleeding or peptic ulcer, Charlson index, statin use, other antiplatelet drugs use, ACE inhibitors or angiotensin receptor blockers use, calcium channel blockers use and diuretics use during the follow-up period. Stratified analysis for MACE was conducted to clarify whether baseline characteristics would influence the association between antiplatelet treatment strategy and outcomes. The data 
were processed with the SAS statistical software, V.9.2 (SAS Institute Inc, Cary, North Carolina, USA) and the Statistical Package for the Social Sciences, V.17.0 (SPSS Inc, Chicago, Illinois, USA). A two sided $\mathrm{p}$ value $<0.05$ was considered to be statistically significant.

\section{RESULTS}

Among 3862 patients receiving aspirin before the index ischaemic stroke and receiving either aspirin or clopidogrel after index stroke during the follow-up period, 1623 were excluded due to a medication possession ratio $<80 \%$, or clopidogrel or aspirin not being prescribed within 30 days of a prespecified end point. Also, 355 patients were excluded due to history of atrial fibrillation, valvular heart disease or coagulopathy. Therefore, 1884 patients were included in our final analysis. There were no significant differences in baseline characteristics (eg, age, sex and Charlson index score) between included vs excluded patients.

Among study-eligible patients, the mean age was 71.1 \pm 10.0 years old and $40 \%$ were women. Characteristics of the participants at baseline and during follow-up period by different types of antiplatelet agents are shown in table 1. The daily aspirin dose before index stroke was not different between groups (101.4 mg vs $100.9 \mathrm{mg}$ ) and the average daily dose was $100.9 \mathrm{mg}$ for aspirin vs $74.6 \mathrm{mg}$ for clopidogrel during the follow-up period. The baseline characteristics between the two groups were not significantly different except that patients receiving clopidogrel were more likely to have gastrointestinal bleeding or peptic ulcer, likely because peptic ulcer is an indication for clopidogrel use under the Taiwan National Health Insurance Bureau reimbursement policy, that is, treatment confounding by indication. Patients receiving clopidogrel were more likely to use statins and diuretics during the follow-up period.

During the mean follow-up of 2.4 years, there were 661 MACE and 601 recurrent strokes. Kaplan-Meier curves suggested clopidogrel, as compared to aspirin, reduced the hazards of MACE (figure 1). For MACE, the annual event rate was $9.9 \%$ in clopidogrel group and $15.8 \%$ in aspirin group. For recurrent stroke, the annual event rate was $8.8 \%$ in clopidogrel group and $14.5 \%$ in aspirin group. Compared to aspirin, clopidogrel was associated with a significantly lower occurrence of future MACE (adjusted $\mathrm{HR}=0.54,95 \%$ CI 0.43 to $0.68, \mathrm{p}<0.001$ ) and recurrent stroke (adjusted HR $=0.54$, $95 \%$ CI 0.42 to $0.69, \mathrm{p}<0.001$ ) after adjustment of relevant covariates. For the secondary end points, the pattern of benefit for clopidogrel users was consistent across several end points, including ischaemic stroke (adjusted HR $=0.55,95 \%$ CI 0.43 to $0.71, \mathrm{p}<0.001$ ), intracranial haemorrhage (adjusted $\mathrm{HR}=0.40,95 \%$ CI 0.17 to $0.97, \mathrm{p}=0.041$ ), and composite of MACE and allcause mortality (adjusted $\mathrm{HR}=0.66,95 \%$ CI 0.55 to $0.78, \mathrm{p}<0.001)$. The risk of all-cause mortality was not different between clopidogrel and aspirin users (adjusted HR=0.97, 95\% CI 0.73 to $1.30, \mathrm{p}=0.853$; table 2). The benefit of clopidogrel was consistent across eight subgroups of baseline characteristics in stratified analysis for future MACE (figure 2).

\section{DISCUSSION}

The 'breakthrough' ischaemic cerebrovascular event in a patient on aspirin is a common scenario frequently encountered by clinicians caring for patients with stroke. Strategies for instituting an antithrombotic regimen to prevent future vascular events in such patients vary widely, largely because there is no dedicated clinical trial evidence to guide practitioners. Few patient registries have the scale, relevant antiplatelet information, or long term follow-up assessment capacity to provide insights into this issue. On the basis of the Taiwan NHIRD, we found, in the event of stroke while on aspirin, switching to clopidogrel is associated with fewer vascular events and fewer recurrent strokes. While these observational data can only be seen as suggestive, the current results may provide clinicians modest evidencebased guidance while they wait for additional data from randomised controlled trials of antithrombotic regimens vs aspirin reinitiation among aspirin treatment failures.

Currently, clopidogrel, aspirin and aspirin plus extended-release dipyridamole are recommended as initial first-line options in preventing recurrent stroke. ${ }^{8}$ Indeed, clinical trials suggest that aspirin plus extendedrelease dipyridamole has superior efficacy to aspirin monotherapy, ${ }^{14}$ and clopidogrel appears to have similar effects on secondary stroke prevention when compared to aspirin plus extended-release dipyridamole. ${ }^{15}$ While there have been no dedicated head to head trials of clopidogrel vs aspirin among patients with ischaemic stroke, based on the aforementioned clinical trial data, one could indirectly infer that clopidogrel may be better than aspirin for secondary stroke prevention in patients with ischaemic stroke overall. Also, greatest platelet inhibitory effect of clopidogrel is found in people with the least inhibition of platelet aggregation by aspirin. ${ }^{16}$ As such it is conceivable that clopidogrel may confer the greatest benefit for patients with aspirin treatment failure.

We found patients receiving aspirin, as compared to clopidogrel, tended to take another antiplatelet agent together and had higher risk of intracranial haemorrhage. This finding is consistent with a recent meta-analysis and the PRoFESS trial showing that when compared to clopidogrel monotherapy, long-term aspirin-based dual-antiplatelet therapy is linked to higher risk of intracranial haemorrhage among patients with ischaemic stroke. ${ }^{15} 17$ Additionally, in the CAPRIE trial, clopidogrel, as compared to aspirin, was associated with a non-significant number of intracranial haemorrhage events among a cohort of patients at high risk for recurrent ischaemic events. ${ }^{18}$

A post hoc analysis of patients with aspirin failure and recent lacunar stroke from the Secondary Prevention of Small Subcortical Strokes Trial (SPS3) cohort suggested 
Table 1 Characteristics of patients at baseline and during the follow-up period according to antiplatelet agents

\begin{tabular}{lllr}
\hline Demographic characteristic & Clopidogrel (N=384) & Aspirin (N=1500) & p Value \\
\hline Men, $n(\%)$ & $229(59.6 \%)$ & $895(59.7 \%)$ & 0.991 \\
Age, years, mean \pm SD & $70.8 \pm 9.5$ & $71.1 \pm 10.2$ & 0.574 \\
Interval from index stroke to enrolment, day, median (IQR) & $18(13-32)$ & $16(11-26)$ & 0.113 \\
Comorbidity, $n(\%)$ & & & \\
$\quad$ Hypertension & $220(57.3 \%)$ & $780(52.0 \%)$ & 0.064 \\
Diabetes mellitus & $169(44.0 \%)$ & $736(49.1 \%)$ & 0.077 \\
Ischaemic heart disease & $64(16.7 \%)$ & $284(18.9 \%)$ & 0.307 \\
Prior stroke/TIA & $87(22.7 \%)$ & $280(18.7 \%)$ & 0.078 \\
Hyperlipidaemia & $78(20.3 \%)$ & $327(21.8 \%)$ & 0.527 \\
GI bleeding/peptic ulcer & $72(18.8 \%)$ & $39(2.6 \%)$ & $<0.001$ \\
Charlson index & $0.8 \pm 0.9$ & $0.8 \pm 1.0$ & 0.774 \\
Medication during the follow-up period & & & 0.022 \\
Statin & $170(44.3 \%)$ & $568(37.9 \%)$ & 0.055 \\
Other antiplatelet drugs* & $22(5.7 \%)$ & $131(8.7 \%)$ & 0.391 \\
ACEI/ARB & $160(41.7)$ & $589(39.3)$ & 0.754 \\
CCB & $207(53.9)$ & $822(54.8)$ & 0.043 \\
Diuretics & $84(21.9)$ & $261(17.4)$ &
\end{tabular}

*Dipyridamole, ticlopidine, cilostazole, or aspitin+dipyridamole.

ACEl: ACE inhibitors; ARB, angiotensin receptor blockers; CCB, calcium channel blockers; GI, gastrointestinal; TIA, transient ischaemic attack.

the addition of clopidogrel did not result in reduction of vascular events vs continuing aspirin only. ${ }^{19}$ Several differences exist between these two cohorts. First, the exact dosage and duration of aspirin use before the index stroke were not known in SPS3 cohort but all participants in our cohort were receiving aspirin for more than 30 days with average dose of $101.3 \mathrm{mg}$ /day at the time of the index stroke. Second, the daily dose of aspirin was $325 \mathrm{mg}$ in SPS3 vs $100.9 \mathrm{mg}$ in the current cohort during study period. Third, SPS3 was conducted in Western countries and the current study was conducted in an Asian country. Asian patients with stroke have higher possibility of intracranial stenosis ${ }^{20}$ and a

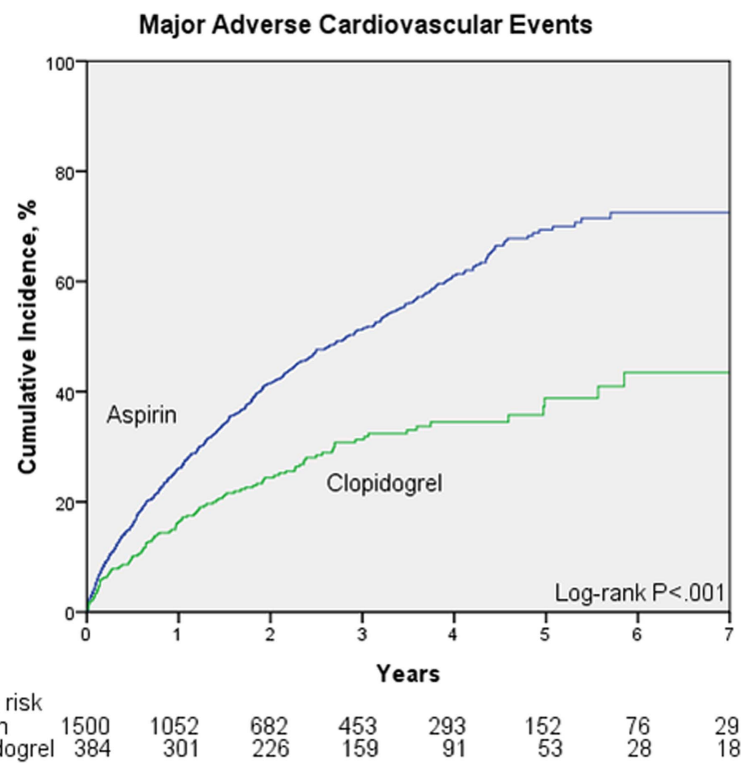

Figure 1 Kaplan-Meier curves for major adverse cardiovascular events among clopidogrel and aspirin groups. study suggested that adding clopidogrel along with aspirin is more effective than aspirin alone in reducing microembolic signals in people with intracranial symptomatic stenosis. ${ }^{21}$

This study has several limitations. First, it is a retrospective cohort study and reasons for using one specific kind of antiplatelet therapy are not well known in this cohort study. Second, information on a few established stroke risk factors, for example, smoking and blood pressure levels during the follow-up period, are not provided in NHIRD. However, these limitations were not likely to greatly bias the overall results. Third, ischaemic stroke type is not provided directly in the NHIRD. Fourth, several patients were excluded from the final analysis due to the nature of the study question and our strict inclusion criteria. Our strict inclusion criteria were driven largely by a desire to exclude patients with poor drug adherence, since such a situation may have confounded our ability to properly address the study question. Also, there were no significant differences in baseline characteristics between included vs excluded patients. Fifth, some non-steroidal anti-inflammatory drugs (NSAIDs), such as ibuprofen, may compete with aspirin for the cyclo-oxygenase 1 binding site and significantly interfere with the antiplatelet activity of aspirin. ${ }^{22}$ We did not explore the impact of NSAIDs use for the current study because the NSAIDs were readily available outside the prescription, and the exact dose and duration of NSAIDs use were difficult to standardise. It is not possible that use of NSAIDs, which is more likely to interfere with antiplatelet activity of aspirin but not with that of clopidogrel, was a significant determinant of higher recurrent vascular events in aspirin group. Sixth, we did not adjust for socioeconomic status of patients because the link between data from the NHIRD and information of socioeconomic status, such as income, is 
Table 2 Occurrence of primary and secondary end points and unadjusted and adjusted HRs by clopidogrel vs aspirin

\begin{tabular}{|c|c|c|c|c|c|c|}
\hline & $\begin{array}{l}\text { Clopidogrel, } \\
\mathrm{N}=384\end{array}$ & $\begin{array}{l}\text { Aspirin, } \\
N=1500\end{array}$ & $\begin{array}{l}\text { Unadjusted HR } \\
(95 \% \mathrm{Cl})\end{array}$ & p Value & $\begin{array}{l}\text { Adjusted HR } \\
(95 \% \mathrm{Cl})\end{array}$ & p Value \\
\hline \multicolumn{7}{|l|}{ Primary outcome } \\
\hline MACE & $91(23.7 \%)$ & $570(38.0 \%)$ & $0.54(0.43$ to 0.68$)$ & $<0.001$ & $0.54(0.43$ to 0.68$)$ & $<0.001$ \\
\hline \multicolumn{7}{|l|}{ Secondary outcomes } \\
\hline Any recurrent stroke & $81(21.1 \%)$ & $520(34.7 \%)$ & 0.53 (0.42 to 0.67$)$ & $<0.001$ & $0.54(0.42$ to 0.69$)$ & $<0.001$ \\
\hline Ischaemic stroke & $75(19.5 \%)$ & $470(31.3 \%)$ & $0.54(0.43$ to 0.70$)$ & $<0.001$ & $0.55(0.43$ to 0.71$)$ & $<0.001$ \\
\hline $\begin{array}{l}\text { Intracranial } \\
\text { haemorrhage }\end{array}$ & $6(1.6 \%)$ & $50(3.3 \%)$ & $0.41(0.18$ to 0.96$)$ & 0.041 & 0.40 (0.17 to 0.97$)$ & 0.041 \\
\hline Fatal stroke & $10(2.6 \%)$ & $52(3.5 \%)$ & $0.71(0.36$ to 1.40$)$ & 0.327 & 0.76 (0.38 to 1.53$)$ & 0.443 \\
\hline Myocardial infarction & $10(2.6 \%)$ & $50(3.3 \%)$ & 0.65 (0.33 to 1.28$)$ & 0.208 & 0.50 (0.24 to 1.05$)$ & 0.067 \\
\hline All-cause mortality & $64(16.7 \%)$ & $229(15.3 \%)$ & 0.93 (0.71 to 1.23$)$ & 0.618 & 0.97 (0.73 to 1.30$)$ & 0.853 \\
\hline $\begin{array}{l}\text { MACE+ all-cause } \\
\text { mortality }\end{array}$ & $155(40.4 \%)$ & $799(53.3 \%)$ & $0.66(0.55$ to 0.78$)$ & $<0.001$ & $0.66(0.55$ to 0.78$)$ & $<0.001$ \\
\hline
\end{tabular}

not allowed in Taiwan. Seventh, patients treated with clopidogrel received lipid-lowering therapy more frequently, but we do not know the exact reason for this. On one hand, it should be pointed out that all included patients had roughly similar compliance since medication possession ratios were $>80 \%$. On the other hand, although there is nationwide regulation of antiplatelet drug prescriptions, it is not inconceivable that some doctors who were more willing to use the antiplatelet drug with higher cost (clopidogrel) were also more inclined to prescribe statin drugs. Finally, our cohort included only Asian patients and the generalisability of the findings to other races is unknown. Future studies will need to include non-Asian patients.
As has been emphasised in the literature, patients who have an ischaemic stroke while taking aspirin need detailed work up to identify the mechanism of their event. ${ }^{823}$ Many of these mechanisms will have a specific indicated therapy, such as carotid endarterectomy or stenting for symptomatic carotid stenosis, anticoagulation for atrial fibrillation and haemodynamic management for collateral failure. If platelet aggregation is determined to be a likely contributing factor to the event, the observational data in our study suggest that, among patients with ischaemic stroke who experience a stroke while on aspirin, that is, the so-called 'aspirin treatment failures', initiation of clopidogrel may be a better long-term choice than reinitiation of aspirin for

\begin{tabular}{|c|c|c|c|c|c|}
\hline Subgroups & No. of patients & $\begin{array}{l}\text { Favors Clopidogrel } \\
\text { therapy }\end{array}$ & $\begin{array}{r}\text { Favors Aspirin } \\
\text { therapy }\end{array}$ & $\begin{array}{c}\text { HR } \\
(95 \% \mathrm{CI})\end{array}$ & $P$ value \\
\hline \multicolumn{6}{|l|}{ Age, years } \\
\hline$<65$ & 450 & $\longrightarrow \square$ & + & $0.74(0.45-1.21)$ & 0.226 \\
\hline $65-75$ & 688 & $\rightarrow \square$ & & $0.42(0.30-0.61)$ & $<0.001$ \\
\hline$\geq 75$ & 746 & $\square$ & & $0.63(0.42-0.93)$ & 0.021 \\
\hline \multicolumn{6}{|l|}{ Gender } \\
\hline Male & 1124 & $\rightarrow \square-$ & & $0.59(0.44-0.79)$ & $<0.001$ \\
\hline Female & 760 & $\square \square$ & & $0.45(0.30-0.66)$ & $<0.001$ \\
\hline \multicolumn{6}{|c|}{ Hypertension } \\
\hline Yes & 1000 & $\square$ & & $0.64(0.47-0.87)$ & 0.004 \\
\hline No & 884 & $-\square-$ & & $0.43(0.29-0.62)$ & $<0.001$ \\
\hline \multicolumn{6}{|l|}{ Diabetes } \\
\hline Yes & 905 & $-\square$ & & $0.43(0.30-0.62)$ & $<0.001$ \\
\hline No & 979 & $\longrightarrow \square$ & & $0.66(0.49-0.90)$ & 0.008 \\
\hline \multicolumn{6}{|c|}{ Myocardial Infarction } \\
\hline Yes & 348 & $\square$ & - & $0.66(0.39-1.12)$ & 0.124 \\
\hline No & 1536 & $-\square-$ & & $0.52(0.40-0.67)$ & $<0.001$ \\
\hline \multicolumn{6}{|l|}{ Prior Stroke } \\
\hline Yes & 367 & $\square \square$ & & $0.55(0.35-0.87)$ & 0.010 \\
\hline No & 1517 & $\rightarrow \square$ & & $0.54(0.42-0.71)$ & $<0.001$ \\
\hline \multicolumn{6}{|c|}{ GI bleeding/ peptic ulcer } \\
\hline Yes & 111 & $\longrightarrow$ & - & $0.56(0.24-1.31)$ & 0.181 \\
\hline No & 1773 & $-\square$ & & $0.55(0.43-0.70)$ & $<0.001$ \\
\hline \multicolumn{6}{|c|}{ Hyperlipidemia } \\
\hline Yes & 405 & $\longrightarrow$ & - & $0.64(0.38-1.08)$ & 0.093 \\
\hline \multirow[t]{2}{*}{ No } & 1479 & $\rightarrow-$ & & $0.51(0.40-0.67)$ & $<0.001$ \\
\hline & & 0.5 & 1.5 & & \\
\hline
\end{tabular}

Figure 2 Stratified analysis for future adjusted risks of major adverse cardiovascular events according to baseline characteristics (clopidogrel vs aspirin). 
future vascular risk reduction. Still, the results should be interpreted in the light of the several limitations as described above. Before considering dedicated randomised clinical trials of clopidogrel initiation vs aspirin reinitiation among patients with ischaemic stroke, prospective cohort studies should explore this issue utilising more precise information on the underlying mechanism of the index stroke and treatment of post-stroke risk factors.

\section{Author affiliations}

${ }^{1}$ Department of Neurology, Chang Gung University College of Medicine, Chang Gung Memorial Hospital, Chiayi Branch, Puzi, Taiwan

${ }^{2}$ Department of Neurology, Stroke Center, Geffen School of Medicine, University of California, Los Angeles, California, USA

${ }^{3}$ Department of Physical Therapy and Assistive Technology, National YangMing University, Taipei, Taiwan

${ }^{4}$ Department of Neurology, Chang Gung University College of Medicine, Chang Gung Memorial Hospital, Kaohsiung Branch, Kaohsiung, Taiwan ${ }^{5}$ Department of Neurology, Chang Gung University College of Medicine, Chang Gung Memorial Hospital, Linkou Branch, Taoyuan, Taiwan

${ }^{6}$ Department of Healthcare Management, Chang Gung University, Taoyuan, Taiwan

${ }^{7}$ Department of Neurosciences, Medical University of South Carolina, Charleston, South Carolina, USA

Contributors $M L$ and $Y-L W$ were involved in the acquisition of data; $M L, Y-L W$, $\mathrm{JLS}$ and $\mathrm{BO}$ were involved in the analysis and interpretation of the data; $\mathrm{ML}$ and $B 0$ were involved in the drafting of the manuscript; $M L, Y-L W, J L S, H-C L, J-D L$, $\mathrm{K}-\mathrm{CC}, \mathrm{C}-\mathrm{YW}, \mathrm{T}-\mathrm{HL}, \mathrm{H}-\mathrm{HW}$, NMR and BO were involved in the critical revision of the manuscript for important intellectual content; YLW was involved in the statistical analysis; ML, H-CL, J-DL, K-CC, C-YW, T-HL and H-HW were involved in obtaining of funding; JLS and BO were involved in the study supervision.

Funding The National Health Research Institute provided access to the National Health Insurance Research Database (NHIRD). This work was supported by grants from Chang Gung Memorial Hospital and National Science Council, Taiwan (CMRPG6B0111, 6B0112 and NSC-102-2628-B-182-012). This study is based on data from the NHIRD provided by the Bureau of National Health Insurance, Department of Health and managed by the NHIRD, Taiwan.

Competing interests None.

Ethics approval This study has been approved by the institutional review board of Chang Gung Memorial Hospital, Chiayi, Taiwan.

Provenance and peer review Not commissioned; externally peer reviewed.

Data sharing statement No additional data are available.

Open Access This is an Open Access article distributed in accordance with the Creative Commons Attribution Non Commercial (CC BY-NC 4.0) license, which permits others to distribute, remix, adapt, build upon this work noncommercially, and license their derivative works on different terms, provided the original work is properly cited and the use is non-commercial. See: http:// creativecommons.org/licenses/by-nc/4.0/

\section{REFERENCES}

1. Eikelboom JW, Hankey GJ. Aspirin resistance: a new independent predictor of vascular events? J Am Coll Cardiol 2003;41:966-8.

2. Hennekens $\mathrm{CH}$, Schror K, Weisman $\mathrm{S}$, et al. Terms and conditions: semantic complexity and aspirin resistance. Circulation 2004;110:1706-8.

3. Davies MJ. Stability and instability: two faces of coronary atherosclerosis. The Paul Dudley white lecture 1995. Circulation 1996;94:2013-20.

4. Halcox JP, Schenke WH, Zalos G, et al. Prognostic value of coronary vascular endothelial dysfunction. Circulation 2002;106:653-8.

5. Ajjan R, Grant PJ. Coagulation and atherothrombotic disease. Atherosclerosis 2006;186:240-59.

6. Watson T, Shantsila E, Lip GY. Mechanisms of thrombogenesis in atrial fibrillation: Virchow's triad revisited. Lancet 2009;373:155-66.

7. Lee M, Ovbiagele B. Vascular events after stroke: Terutroban fails to perform. Lancet 2011;377:1980-2.

8. Furie KL, Kasner SE, Adams RJ, et al. Guidelines for the prevention of stroke in patients with stroke or transient ischemic attack: a guideline for healthcare professionals from the American Heart Association/ American Stroke Association. Stroke 2011;42:227-76.

9. Cheng $\mathrm{CL}, \mathrm{Kao} \mathrm{YH}$, Lin SJ, et al. Validation of the national health insurance research database with ischemic stroke cases in Taiwan. Pharmacoepidemiol Drug Saf 2011;20:236-42.

10. Goldstein LB, Samsa GP, Matchar DB, et al. Charlson index comorbidity adjustment for ischemic stroke outcome studies. Stroke 2004;35:1941-5.

11. Wong MC, Tam WW, Cheung CS, et al. Drug adherence and the incidence of coronary heart disease-and stroke-specific mortality among 218,047 patients newly prescribed an antihypertensive medication: a five-year cohort study. Int J Cardiol 2013;168:928-33.

12. Garcia Rodriguez LA, Cea Soriano L, Hill C, et al. Increased risk of stroke after discontinuation of acetylsalicylic acid: a UK primary care study. Neurology 2011;76:740-6.

13. Wu CY, Chen YJ, Ho HJ, et al. Association between nucleoside analogues and risk of hepatitis $B$ virus-related hepatocellular carcinoma recurrence following liver resection. JAMA 2012;308:1906-14.

14. Halkes PH, van Gijn J, Kappelle LJ, et al. Aspirin plus dipyridamole versus aspirin alone after cerebral ischaemia of arterial origin (ESPRIT): Randomised controlled trial. Lancet 2006;367:1665-73.

15. Sacco RL, Diener HC, Yusuf S, et al. Aspirin and extended-release dipyridamole versus clopidogrel for recurrent stroke. $N$ Engl J Med 2008;359:1238-51.

16. Eikelboom JW, Hankey GJ, Thom J, et al. Enhanced antiplatelet effect of clopidogrel in patients whose platelets are least inhibited by aspirin: a randomized crossover trial. J Thromb Haemost 2005;3:2649-55.

17. Lee M, Saver JL, Hong KS, et al. Risk-benefit profile of long-term dual-versus single-antiplatelet therapy among patients with ischemic stroke: a systematic review and meta-analysis. Ann Intern Med 2013;159:463-70.

18. CAPRIE steering committee. A randomised, blinded, trial of clopidogrel versus aspirin in patients at risk of ischaemic events (CAPRIE). Lancet 1996;348:1329-39.

19. Cote R, Zhang Y, Hart RG, et al. ASA failure: does the combination ASA/clopidogrel confer better long-term vascular protection? Neurology 2014;82:382-9.

20. Wong LK. Global burden of intracranial atherosclerosis. Int J Stroke 2006;1:158-9.

21. Wong $\mathrm{KS}$, Chen $\mathrm{C}, \mathrm{Fu}$ J, et al. Clopidogrel plus aspirin versus aspirin alone for reducing embolisation in patients with acute symptomatic cerebral or carotid artery stenosis (CLAIR study): a randomised, open-label, blinded-endpoint trial. Lancet Neurol 2010;9:489-97.

22. Saxena A, Balaramnavar VM, Hohlfeld T, et al. Drug/drug interaction of common NSAIDs with antiplatelet effect of aspirin in human platelets. Eur J Pharmacol 2013;721:215-24.

23. Ali LK, Saver JL. The ischemic stroke patient who worsens: New assessment and management approaches. Rev Neurol Dis 2007;4:85-91. 